Monatsschr Kinderheilkd 2009 • 157:1096-1097 DOI 10.1007/s00112-009-2116-5

Online publiziert: 6. November 2009

(c) Springer Medizin Verlag 2009

\author{
V. Mall ${ }^{1} \cdot$ F. Heinen ${ }^{2} \cdot$ U. Michaelis ${ }^{1}$ \\ ${ }^{1}$ Klinik II, Neuropädiatrie und Muskelerkrankungen, Zentrum für Kinderheilkunde \\ und Jugendmedizin,Universitätsklinikum Freiburg \\ ${ }^{2}$ Abteilung Pädiatrische Neurologie und Entwicklungsneurologie, Kinderklinik, \\ Dr. von Haunersches Kinderspital, Ludwig-Maximilians-Universität München
}

\title{
Klassifikation der motorischen Fähigkeiten von Kindern mit Zerebralparese
}

keiten eingesetzt, deren völlig uneinheitliche und unscharfe Anwendung die kanadische Arbeitsgruppe um Peter Rosenbaum zur Entwicklung des GMFCS veranlasste. Rosenbaum et al. [8] kam zugute, dass sie auf ein selbstentwickeltes Evaluationsinstrument zurückgreifen konnten, das GMFM („gross motor function measure"). Dabei handelt es sich um ein weltweit benutztes, kriterienbezogenes Beobachtungsinstrument, welches für Kinder mit Zerebralparese entwickelt und inzwischen auch für Kinder mit Morbus Down [9] und für Kinder nach SchädelHirn-Trauma [3] validiert wurde.

Anwendungshinweise. Das GMFCS basiert auf selbstinitiierten Bewegungen unter besonderer Beachtung der Sitzfähigkeit, des Transfers und der Mobilität. Bei der Festlegung einer 5-Stufen-Klassifikation wurde v. a. darauf geachtet, dass die Unterschiede zwischen den definierten Stufen für das tägliche Leben bedeutsam sind. Sie basieren auf funktionellen Einschränkungen, dem Gebrauch von Gehhilfen (wie Rollator, Unterarmgehstützen oder Gehstöcken) oder von mobilen Hilfsmitteln (Rollstuhl). Die Qualität der funktionellen Fähigkeiten wird dabei nur bedingt berücksichtigt. Die Unterschiede zwischen den Stufen I und II sind nicht so deutlich wie die zwischen den anderen Stufen, dies gilt besonders für Kinder im Alter von unter 2 Jahren.

Entwicklung der GMFCS. Bis zur Entwicklung des GMFCS im Jahre 1997 wurden die Begriffe „leicht, mittel und schwer“ zur Beschreibung der motorischen Fähig-

\section{Unterschiede zwischen Stufe I und II.} Verglichen mit Kindern/Jugendlichen der Stufe I haben Kinder/Jugendliche der Stu- fe II Einschränkungen beim Zurücklegen weiter Strecken und Schwierigkeiten mit der Balance; sie können eine Gehhilfe beim Erlernen des Laufens benötigen. Bei langen Strecken außerhalb des Hauses oder in der Umgebung können sie auf so genannte rollende Fortbewegung angewiesen sein. Sie benötigen ein Treppengeländer, um Treppen hinauf und herunter zu gehen und sind beim Rennen und Hüpfen nicht so geschickt.

Das erweiterte GMFCS [7] berücksichtigt Jugendliche von 12-18 Jahren und basiert auf dem Konzept der Internationalen Klassifikation für Funktionsfähigkeit, Behinderung und Gesundheit („international classification of functioning, disability and health", ICF) der Weltgesundheitsorganisation (WHO).

\section{Schwerpunkt der GMFCS ist die Bestimmung der Stufe der aktuellen motorischen Möglichkeiten}

Die Autoren des GMFCS ermutigten die Anwender, darauf zu achten, welchen Einfluss Umfeld und persönliche Faktoren auf die Tätigkeiten haben, die sie bei Kindern und Jugendlichen beobachten oder die ihnen berichtet werden. Der Schwerpunkt der GMFCS ist die Bestimmung der Stufe, die am besten die aktuellen Möglichkeiten und Grenzen in der Körpermotorik des Kindes oder Jugendlichen widerspiegelt. Maßgebend ist die gewöhnliche Leistung zu Hause, in der Schule und bei gesellschaftlichen Aktivitäten (d. h. was die Kinder/Jugendlichen tun) und nicht 
Tab. 1 Darstellung der Klassifikation der motorischen Fähigkeiten

\begin{tabular}{|ll}
\hline Stufe & Motorische Fähigkeiten \\
\hline I & Geht ohne Einschränkungen \\
\hline II & Geht mit Einschränkungen \\
\hline III & Geht mit Benutzung einer Gehhilfe \\
IV & Selbstständige Fortbewegung eingeschränkt, es kann ein E-Rollstuhl benutzt werden \\
V & Wird in einem Rollstuhl gefahren \\
E-Rollstuhl Elektrorollstuhl
\end{tabular}

die bestmöglichste Fähigkeit, die die Kinder/Jugendlichen tun könnten. Es ist deshalb wichtig, lediglich die aktuelle Körpermotorik einzustufen und keine Einschätzungen über die Qualität der Bewegung vorzunehmen oder Prognosen über die zukünftigen Verbesserungsmöglichkeiten abzugeben. Die Bezeichnung jeder Stufe erfolgt nach der Fortbewegungsmethode, die für die Ausführung nach dem 6. Lebensjahr charakteristisch ist. Die Beschreibung der funktionellen Möglichkeiten und Einschränkungen ist für jede Altersklasse absichtlich breit angelegt; in diesem Sinne ist es nicht vorgesehen, alle Aspekte der motorischen Funktion eines individuellen Kindes/Jugendlichen zu beschreiben. Ein Kind mit einer Hemiparese z. B., das nicht auf Händen und Knien krabbeln kann, aber sonst der Beschreibung von Stufe I entspricht (d. h. es kann sich in den Stand ziehen und laufen), würde in die Stufe I eingeteilt werden. Die Klassifikation benutzt eine Ordinalskala, bei der der Abstand zwischen den einzelnen Stufen nicht konstant ist und Kinder und Jugendliche mit Zerebralparesen nicht gleichmäßig auf die 5 Stufen verteilt sind. Es ist den Autoren bewusst, dass die Manifestation der Körpermotorik vom Alter abhängig ist, dies gilt insbesondere für das Kleinkindalter und die frühe Kindheit.

Für jede Stufe werden eigene Beschreibungen für die unterschiedlichen Altersgruppen gegeben [10]. Bei Frühgeborenen sollte bis zum 2. Lebensjahr das korrigierte Lebensalter benutzt werden. Die Beschreibungen für die 6- bis 12-Jährigen und die 12- bis 18-Jährigen berücksichtigen den potenziellen Einfluss von Umgebungsfaktoren (z. B. Entfernungen in der Schule und der Umgebung) und von persönlichen Faktoren (z. B. Energieverbrauch und soziale Präferenzen) auf die Fortbewegungsmöglichkeiten. Es wurde versucht, die motorischen Fähigkeiten der Kinder und Jugendlichen in den Vor- dergrund zu stellen und nicht ihre Beeinträchtigungen. Als allgemeines Prinzip gilt deshalb, dass Kinder und Jugendliche, die die motorischen Fähigkeiten einer bestimmten Stufe erfüllen, in diese oder eine darüber liegende Stufe eingeordnet werden. Entsprechend sollten Kinder und Jugendliche, die die motorischen Fähigkeiten einer Stufe nicht erfüllen, eher einer darunter liegenden Stufe zugeordnet

\section{Ausblick}

Das GMFCS hat eine definierte, international einheitliche und fachübergreifende Sprachregelung für die Klassifikation motorischer Fähigkeiten von Kinder und Jugendliche mit Zerebralparesen geschaffen. Der Anspruch des Instrumentes geht jedoch darüber hinaus. So weisen die Autoren darauf hin, dass der Einfluss von Umweltfaktoren bei der Klassifikation explizit berücksichtigt werden soll.

\section{(7) Das GMFCS-Instrument ist Explorationsgrundlage weiterer zentraler Bereiche der CP}

Das Instrument ist als Grundlage und Türöffner zur Exploration weiterer zentraler Bereiche der Zerebralparese zu sehen. So konnten mit Hilfe des GMFCS der Zusammenhang zwischen Lebensqualität und motorischen Fähigkeiten und die Relevanz des familiären Kontextes untersucht werden [7].

\section{Korrespondenzadresse \\ Prof. Dr. V. Mall}

Klinik II, Neuropädiatrie und Muskelerkrankungen, Zentrum für Kinderheilkunde und Jugendmedizin, Universitätsklinikum Freiburg Mathildenstraße 1, 79106 Freiburg volker.mall@uniklinik.freiburg.de

Interessenkonflikt. Keine Angaben werden (• Tab. 1).

\section{Literatur}

1. Cans C, Dolk H, Platt MJ et al (2007) Recommendations from the SCPE collaborative group for defining and classifying cerebral palsy. Dev Med Child Neurol [Suppl] 1099:35-38

2. Heinen F, Schröder AS, Döderlein L et al (2009) Grafikgestützter Konsensus für die Behandlung von Bewegungsstörungen bei Kindern mit bilateral spastischer Zerebralparese (BSCP). Therapiekurven - CP Motorik. Monatsschr Kinderheilkd 157:789-794

3. Linder-Lucht $M$, Othmer V, Walther M et al (2007) Validation of the gross motor function measure for use in children and adolescents with traumatic brain injuries. Gross Motor Function Measure-Traumatic Brain Injury Study Group. Pediatrics 120(4): e880-886

4. Palisano R, Rosenbaum PL, Walter S et al (1997) Development and reliability of a system to classify gross motor function in children with cerebral palsy. Dev Med Child Neurol 39(4):214-223

5. Palisano RJ, Hanna SE, Rosenbaum PL, Tieman B (2009) Probability of walking, wheeled mobility, and assisted mobility in children and adolescents with cerebral palsy. Dev Med Child Neurol Aug 27. [Epub ahead of print]

6. Rosenbaum PL, Walter SD, Hanna SE et al (2002) Prognosis for gross motor function in cerebral palsy: creation of motor development curves. JAMA 288(11):1357-1363

7. Rosenbaum PL, Palisano RJ, Bartlett DJ et al (2008) Development of the gross motor function classification system for cerebral palsy. Dev Med Child Neurol 50(4):249-253

8. Russell DJ, Rosenbaum PL, Cadman DT et al (1989) The gross motor function measure: a means to evaluate the effects of physical therapy. Dev Med Child Neurol 31(3):341-352

9. Russell D, Palisano R, Walter S et al (1998) Evaluating motor function in children with Down syndrome: validity of the GMFM. Dev Med Child Neurol 40(10):693-701

10. Russel D, Rosenbaum P, Avery L et al (2006) GMFM und GMFCS Messung und Klassifikation motorischer Funktionen. Huber, Bern 